\title{
Barriers against required nurse estimation models applying in Iran hospitals from health system experts' point of view
}

Seyed Saeed Tabatabaee ${ }^{1}$, Mahmood Nekoie-Moghadam² ${ }^{2}$ Ali Vafaee-Najar ${ }^{3}$, Mohammad Reza Amiresmaili ${ }^{4}$

${ }^{1}$ Ph.D. Candidate, Department of Health Management, Policy \& Economics, Health Services Management Research Center, Institute for Futures Studies in Health, Kerman University of Medical Sciences, Kerman, Iran

${ }^{2}$ Professor, Environmental Health Engineering Research Center, Health Services Management Department, Kerman University of Medical Sciences, Kerman, Iran

${ }^{3}$ Associate Professor, Health Sciences Research Center, Department of Health Management, School of Health, Mashhad University of Medical Sciences, Mashhad, Iran

${ }^{4}$ Associate Professor, Medical Informatics Research Center, Institute for Futures Studies in Health, Kerman University of Medical Sciences, Kerman, Iran

\section{Type of article: Original}

\begin{abstract}
Introduction: One of the strategies for accessing effective nursing care is to design and implement a nursing estimation model. The purpose of this research was to determine barriers in applying models or norms for estimating the size of a hospital's nursing team.

Methods: This study was conducted from November 2015 to March 2016 among three levels of managers at the Ministry of Health, medical universities, and hospitals in Iran. We carried out a qualitative study using a Colaizzi method. We used semistructured and in-depth interviews by purposive, quota, and snowball sampling of 32 participants (10 informed experts in the area of policymaking in human resources in the Ministry of Health, 10 decision makers in employment and distribution of human resources in treatment and administrative chancellors of Medical Universities, and 12 nursing managers in hospitals). The data were analyzed by Atlas.ti software version 6.0.15.

Results: The following 14 subthemes emerged from data analysis: Lack of specific steward, weakness in attracting stakeholder contributions, lack of authorities trust to the models, lack of mutual interests between stakeholders, shortage of nurses, financial deficit, non-native models, designing models by people unfamiliar with nursing process, lack of attention to the nature of work in each ward, lack of attention to hospital classification, lack of transparency in defining models, reduced nurses available time, increased indirect activity of nurses, and outdated norms. The main themes were inappropriate planning and policymaking in high levels, resource constraints, and poor design of models and lack of updating the model.

Conclusion: The results of present study indicate that many barriers exist in applying models for estimating the size of a hospital's nursing team. Therefore, for designing an appropriate nursing staff estimation model and implementing it, in addition to considering the present barriers, identifying the norm required features may positively impact on norm acceptance and implementation.

Keywords: Qualitative Research, Nurse, Hospital, Model
\end{abstract}

\section{Introduction}

The aim of human resource planning is to assure the existence of the right size and mix of staff in the right time and place (1). Because salaries and benefits of a health staff make up the biggest part of a health budget (2), the attribution of the proper numbers of employee in a health care organizations is essential for achieving optimal

\section{Corresponding author:}

Associate Professor Dr. Mohammad Reza Amiresmaili, Medical Informatics Research Center, Institute for Futures Studies in Health, Kerman University of Medical Sciences, Kerman, Iran.

Tel: +98.3432315147 Fax:+98.3432315221, Email: mohammadreza.amiresmaili@gmail.com

Received: May 01, 2016, Accepted: July 05, 2016, Published: December 2016

iThenticate screening: July 04, 2016, English editing: September 10, 2016, Quality control: October 26, 2016

(C) 2016 The Authors. This is an open access article under the terms of the Creative Commons Attribution-NonCommercialNoDerivs License, which permits use and distribution in any medium, provided the original work is properly cited, the use is non-commercial and no modifications or adaptations are made. 
effectiveness and efficiency (3). To provide efficient services, some strategies such as designing models for staff estimation and distribution are applied. A human resource model in an organization is defined as a way to ensure the availability of a proper and sufficient workforce appropriated to workload and vice versa. The standards of human resources in health care are defined in two levels of expected quantity and quality of required staff (4). The use of an acceptable and practical standard for estimating the workforce is an essential and undeniable fact in health care organizations (5). One of the most important challenges of hospitals in the Iranian health system is inadequate distribution of human resources (6). Zandian et al. evaluated the inequality in distribution of human resources in Ardebil province. The authors concluded that the distribution of human resources and hospital beds is not balanced (7). Sadeghifar et al. assessed the required human resources in hospitals related to Ilam University. The authors concluded that, generally, the studied hospitals lack proper human resources, and the distribution of human resources was not appropriate, either (8). Ghazi Mirsaeid et al. evaluated the distribution of specialized human resource in hospitals of Tehran University of Medical Sciences and found that human resource distribution in hospitals does not follow any special model. Accordingly, development of a plan for appropriate required human resource distribution in the healthcare system of Iran is considered one of the essential steps to promote accountability and fairness in the healthcare reform map (9). Among health staff, nurses are the largest group of professional staff $(10,11)$. Increasing effectiveness and efficiency of nursing care is essential in providing safe care to patients $(12,13)$. Because nursing staff have a crucial role in the quality of patient care and safety of patients, utilization of nursing staff in hospitals has become a major concern (14). Farsi et al. believed that an insufficient number and unequal distribution of nurses is one of the challenges in Iran's health system (15). Generally, the shortage and unequal distribution of nurses are described as the gap between the number of available nurses and number of needed nurses (16). Rossetti and Gaidzinski in their study about estimating nursing staff required in a new hospital, stated that a new hospital faces numerous and complex challenges, estimating the number of required nurses is one of them (17). In Iran a few models for estimating the number of required nurses in hospitals are designed; however, it seems that many managers do not pay attention to these models. The details of two main proposed models in hospitals are described as follows:

1) Allocation of post approach: in this model, for each hospital bed 0.85 nurse staff is considered. Nurse staff included Merton, nurse, nurse aid, operation room operator, and anesthesiologist assistant.

2) Systematic approach: in this model, each ward has an especial coefficient. To calculate the number of required nurses in each ward, the number of beds is multiplied by the coefficient. For example, the coefficient for internal medicine is 0.74 , which means for every two beds 1.5 nurse is needed (6).

Because hospitals are the most important organization employing nurses and because failure to use the nursing staff estimation and distribution models leads to unequal distribution of nurses, which could affect quality and efficiency of services, the aim of the present study was to describe the challenges that hospitals face in using nursing staff estimation models from the perspective of health system experts. We hoped that the results of this study help to identify factors related to unwillingness of hospital managers, human resource manages, and nursing managers in using present models in Iran. We also expect that the results help other researchers in finding solutions in regards to barriers.

\section{Material and Methods}

\subsection{Design}

This study was performed by qualitative methodology with a phenomenological approach. A phenomenological approach tries to reveal the meanings that people experienced in their life (18). The main reason for choosing a phenomenology approach in this study was to better describe the study subject through experiences of informed people considering the less published materials or because of the need for deeper description and explanation of subject (19).

\subsection{Participants and Setting}

The study subjects were selected from three levels of managers in a health system: high-level mangers (from the Ministry of Health), middle-level managers (from Medical Sciences Universities and Provincial Medical Councils), and executive managers (from hospitals). The sampling was performed using the purposive, quota, and snowball methods. The reasons for choosing the purposive, quota, and snowball sampling methods were as follows: for selecting people who have special views about the study subject, faster and easier access to other people who were difficult to be identified by the researcher, and to access more and deeper information (19). Thirty-two subjects were selected from managers who were informed and expert in human resource policymaking in health ministry, were involved in decision making regarding human resources in medical universities, and were nursing managers in 
hospitals. The inclusion criteria were to have at least four years' management experience, knowledge about nursing staff estimation and distribution model, and inclination to participate in the study.

\subsection{Data Collection}

Data were collected through in-depth and semistructured interviews performed by researchers. A literature review on the topic was conducted at first; then two in-depth interviews were performed. Finally, based on findings, an interview guide was designed. The researcher contacted participants by phone to set date and time of interviews. For participants' convenience, the interviews were conducted in their office in a quiet condition. Verbal consent was taken from all participants for recording the interview. The mean duration of interviews was 43 minutes; each session lasted from 40 to 45 minutes. The data collection was performed from November 2015 to March 2016. Data collection continued until data saturation was complete.

\subsection{Data Analysis}

To determine main themes and subthemes, the Colaizzi's method and Atlas.ti software version 6.0.15 were used. Qualitative analysis with Collaizzi method is a suitable method for extracting valid and reliable results from text data, for production of knowledge and new perspectives, to depict the actual data, and to provide practical guidelines (20). The data analysis was performed in a few steps. First, to find the general understanding, the context of interviews was reviewed a few times. The second and fourth authors each separately reread the texts to understand the meanings and then discussed their perceptions with each other in the meetings. In next step, the sentences or phrases directly related to the topic were extracted from interviews. The extracted texts were organized, and then meaningful units were summarized and divided into categories and clusters by the first and the third authors separately. With combining all the extracted results, a full and comprehensive description from the topic was delivered to participants. Although words reliability and validity are related to quantities studies (21), to check data accuracy, four criterions for reliability, confirm ability, and transferability based on the proposed criteria by Lincoln and Gubad were used (22). Research validity was confirmed by reviewing the written interviews by participants and expert teachers. To confirm reliability and confirm ability of study, a report from all the research activities, including data collection, data analysis, and a selection of interviews, was delivered to an expert in qualitative research. For proper information transfer, research details were described carefully to participants with details to clarify the study process.

\subsection{Ethical Considerations}

The research protocol was approved by the ethic committee of Kerman Medical Sciences University (ref. no: 29/217). An introduction letter was taken from the management and information faculty and submitted to participants. The participants received sufficient information about the aim of study, and verbal consent was taken from them. They were assured that the data would be kept confidential and results would be published without name.

\section{Results}

Thirty-two subjects were selected from high-, middle-, and functional-level managers of health care, including 19 men and 13 women. The participants' response rate was $100 \%$. The mean age of participants was $47.3 \pm 6.12$ years, and the mean years of work experience was $23.7 \pm 5.7$. Most of them (37.5\%) had a Ph.D. degree. After interviews and field notes were analyzed, the themes and subthemes of barriers existing in implementing nursing staff estimation model in Iranian hospitals were determined (Table 1).

\subsection{Inappropriate Planning and Policymaking at a High Level}

\subsubsection{Lack of specific steward}

These subthemes indicated that, at the top of the Ministry of Health in Iran, many domains are involved in estimating nursing staff, which means no specific steward officially existed for designing and supervising the implementation of nursing staff estimation model. One of the participants in this regard stated that "presently in development chancellor of Ministry of Health two departments considered they responsible for this matter. These departments are Office of Human Resources Management and Development and Evolution Center. These two offices do not have any communication with each other. This condition is also exited between treatment and nursing chancellors." (p. 5) 
Table 1. Themes and subthemes of this study

\begin{tabular}{|l|l|}
\hline Themes & Subthemes \\
\hline Inappropriate planning and policymaking in high & Lack of specific steward \\
\cline { 2 - 2 } & Weakness in attracting stakeholder contribution \\
\cline { 2 - 2 } & Lack of authorities trust to the models \\
\cline { 2 - 2 } & Lack of mutual interests between stakeholders \\
\hline \multirow{2}{*}{ Resource constraints } & Shortage of nurses \\
\cline { 2 - 2 } & Financial deficit \\
\cline { 2 - 2 } & Non-native models \\
\cline { 2 - 2 } & $\begin{array}{l}\text { Designing models by people unfamiliar with nursing } \\
\text { process }\end{array}$ \\
\cline { 2 - 2 } & Lack of attention to the nature of work in each ward \\
\cline { 2 - 2 } & Lack of attention to hospital classification \\
\cline { 2 - 2 } & Lack of transparency in defining models \\
\hline Lack of updating the model & Reduced nurses available time \\
\cline { 2 - 2 } & Increased indirect activity of nurses \\
\cline { 2 - 2 } & Outdated models \\
\hline
\end{tabular}

\subsubsection{Weakness in attracting stakeholder contribution}

In the opinions of high-level participants, the existence of multiple officials and unofficial models in the country indicated that the important process of model development was performed behind closed doors and lack the participation of stakeholders. One manager said: "I was a member of one of teams developing human resource model in the ministry, but there was no member from nursing filed, when I asked why they replied it is confidential and until design is complete. No one has to know!" (p. 19)

\subsubsection{Lack of authorities' trust to the models}

Selected participants thought that, although different areas are involved in estimating nursing staff in Ministry of Health, these models are not seriously supported by either area. One person stated: "I wish that they had commitment to the models they proposed and implement it, unfortunately they do not accept even their own developed models." (p. 6)

\subsubsection{Lack of mutual interests between stakeholders}

From the perspective of some high-level managers, lack of mutual interest among stakeholders is an important factor leading to failure in decision and policymaking for nursing staff estimation model in hospitals. In this regard, one participant mentioned: "Unfortunately different departments in the health ministry do not have communication with each other and with the Nursing Council. The Nursing Council defends nurses' rights; the human resource manager wants to reduce nursing staff for decreasing the cost. Until a common interest does not exist between them, the designed model is always rejected by one of the stakeholders." (p.7)

\subsection{Resource constraints}

\subsubsection{Shortage of nurses}

A nurse shortage means that in one period the demand for nurse is greater than available nurses. A nurse shortage is not equal to lack of enough nurses and could be related to other factors such as policymaking, culture, economy, organization, and ... conditions. Most subjects in a high level believe that an adequate supply of nurses has a crucial role in better implementation of models. Regarding this, one person said: "I think we do not have enough nurses to implement these models. Unfortunately, implementation of improve productivity law consumed all of our nurses." (p. 18)

\subsubsection{Financial deficit}

Financial deficit, as one of main affecting factors, could induce many limitations in employing human resources. A number of participants of high-level believe that providing the number of needed nurses for hospitals requires a big budget, which is not currently possible considering the economic condition of country. One manager stated: "Look, right now we talked about financial saving, resistive economy, and contractile thoughts, therefore the ministry does not accept standard that has cost." (p. 13) 


\subsection{Poor design of models}

\subsubsection{Non-native models}

Most participants thought the nursing staff estimation models are borrowed from other countries and cannot be accountable in Iran. They believed that type of equipment and facilities in hospitals of different countries are not similar, therefore their nurse estimation models are not applicable here. One participant mentioned: "I think these models are designed in Australia or USA. We do not have even one ward similar to their hospital wards, how we are expected to distribute our nurses with these models?" (p. 9)

\subsubsection{Designing models by people unfamiliar with nursing process}

It is expected that, if individuals familiar with nursing processes participate in designing a model or norm, the model contains the least amount of errors and would be probably better accepted by nurses. In this regard, one manager said: "These models are designed by people who never worked as nurse and are not familiar with hospital and therapy procedures; therefore models are not executable and accountable in real world." (p. 6)

3.3.3. Lack of attention to the nature of work in each ward

Each hospital based on its goals has different wards, and each ward is nominated based on type of hospitalized patients. The type and amount of nursing care is dependent on the type of hospitalized patients in each ward. Most participants from the middle and functional levels believed that inattention to the nursing workload in each ward when designing a model is one of the barriers in implementing the model. "The same way that a physician cannot prescribe one prescription for all of his patients, we cannot consider one nurse to patient ratio for all hospitals beds. The same way each patient need his own prescription, each hospital bed required its especial ratio." (p. 18)

\subsubsection{Lack of attention to hospitals classification}

Some participants in the middle and functional levels thought that the nurse estimation model must be different based on hospital activity and hospital place in health care and referral system. Type of patients admitted into therapeutic hospitals is different from educational hospitals regarding the complexity of offered treatment and required care. In this regard, one manager said: "In a therapeutic hospital, the extreme performed surgery is an appendectomy; however, in an educational hospital, cancer surgery is performed; therefore type of needed nursing care is different between them." (p. 7)

\subsubsection{Lack of transparency in defining models}

Transparency in defining the model is an influential factor for stakeholders in accepting the model. Lack of answer to stakeholders' questions about technical issues in designing the model and lack of strong evidences for defending the design may lead to non-implementation. "When we look for evidences in designing these models we find nothing, it means that no evidences existed to defend these models, no one knows what the base for designing these models is!" (p. 7)

\subsection{Lack of updating the model}

\subsubsection{Reduced nurses' available time}

The availability of staff in a defined period is an important factor in estimating required human resources. Several participants in the functional level believed that changes in laws regarding the nurses' presence in the workplace in recent years has decreased the nurses' availability. "We have a law in country named 'improve productivity,' which deceased the nurses' available time; this law is not foreseen in any designed models." (p. 9)

3.4.2. Increased indirect activities of nurses

Nurses' indirect activities, which are mainly related to documentation, are part of nurses' workload. A large part of these activities are related to plans dictated by the Ministry of Health, including clinical governance, hospital accreditation, and so on. Most participants from the functional level believed that these activities are increased each year without increasing any workforce. One subject said: "Look, we have a few forms in the hospital such as the patient education form, consent form, primary evaluation form, and nutrition evaluation form and so on. All of them have to be filled, each one has 40 questions, and so what should a nurse do? Fill these forms! Take care of the patient! If a nurse is supposed to do this documentation, the number of his patients must decrease." (p. 4)

\subsubsection{Outdated models}

A number of subjects in the middle and functional levels indicated that using the old outdated models due to changes in therapeutic procedures and laws related to nurses' availability is inefficient. One manager stated: "The models belonged to long ago and are not updated. We know that the pace of innovation in medical equipment and type of care is very high; we cannot manage hospital with a model belonged to 10 years ago." (p. 14)

\section{Discussion}

The results of this study indicate that the most important barriers in implementing nursing staff estimation model in hospitals from the perspective of health care experts are lack of specific stewards, weakness in attracting stakeholder 
contribution, lack of authorities' trust in the models, lack of mutual interests between stakeholders, nurse shortage, finical deficit, non-native models, models designed by people unfamiliar with nursing process, lack of attention to the nature of work in each ward, lack of attention to hospitals' classification, lack of transparency in defining models, reduced nurses' availability time, increased indirect activities of nurses, and outdated models. A number of subjects declared that the existence of different administrative domains in the same managerial level in the Ministry of Health, which each domain had power to affect others decisions, can influence the implementation of human resource estimation model. It seems that lack of a specific steward for this area cause challenges in planning and policy making including weakness in attracting stakeholders' contribution. The lack of stakeholder contribution indicated that the designed model is only written on paper and never will be implemented in hospitals because each of stakeholders have power to prevent implementation. This proceeding have made participants believe that the lack of stakeholder contribution in decision-making about models make them unfaithful to the models. Part of findings of this study indicated that the lack of mutual benefits among stakeholders is one of the reasons for their lack of contribution in designing process, which, consequently, leads to their lack of faith and support for model implementation. Biglar showed that lack of agreement about structural steward in planning and policy-making, and lack of attention to stakeholders is one of the most important steward problems in the Iranian health system (23). Lundenburg, in regard to decision-making, believed that decisions made by teams are planned by more knowledge and expertise, and the final decision is more acceptable, which eventually causes members to have more faith to the model implementation (24). Buchan in his study compared the characteristics and early outcome of using nurse-topatient ratios in California (USA) and Victoria (Australia). He stated that one of the biggest challenges in using nursing ratios is lack of support of entire stakeholders (25).

To improve health services, health systems in developed and underdeveloped countries are under pressure by increased population and limited resources. Human resources, as one of limited resources, has a key role in providing health services, quality of care, and cost (26). It seems that nurse shortages, besides prevention of proper implementation of a nursing staff estimation model, can induce other problems such as job dissatisfaction, increased obligatory overtime work, and increased workload. Sadeghifar et al. estimated the number of required nurses in two hospitals of Emam Khomaini and Razi based on a model proposed by the Ministry of Health. The authors found that 18 out of 19 wards in Emam Khomaini hospital and eight out of nine wards in Razi Hospital have less nursing staff than the proposed standard (27). Research has shown that financial deficit in high level is one of the other barriers in implementing nursing staff estimation model in hospitals. Reiter et al. indicated that enforcing the law of minimum nurses in California put considerable financial pressure on some hospitals (28). It seems that the financial deficit in the governmental level will not be solved in the short term. In the six country development plan, which started in March 2016, it is indicted that any employment of manpower with cost, capital, and financial credits is limited to $20 \%$ of executive organization output; in a way that, at the end of program, the total of human resources of executive organizations (with consideration the decrease of staff in regard to performing clause one and natural causes) in comparison with 2015 decreases by at least 15\% (29). Based on study findings, using non-native models is another barrier in implementing nursing staff estimation models. In management science, each phenomena is influenced by social, economic, culture, and similar factors (30). Ignjatovic and Svetlik declared that issues related to each country have an impact on people and organizational behaviors, thus causing differences in performance, techniques, and methods (31). It appears that one effective $\mathrm{n}$ operationalization of models is to tailor the models from other countries with culture, social, economic, and other related issues of the recipient country. In this regard, the background factors that impact models implementation must be identified and explained.

Designing a nursing staff estimation model by people unfamiliar with nursing processes is also a barrier for successful implementation of a model. Designing a human resource estimation model as a tool for estimating the required manpower has particular importance. Therefore, to reduce probability of error and increase model acceptance, experts and process owners must be involved in model designing. Twigg and Duffield in this concern stated that usually nursing staff estimation models such as nurse-to-bed ratio, nurse-to-patient ratio, and nursing hours per patient day are designed by professional groups (32). Identifying the proper and sufficient levels of nursing staff employment is a prerequisite feature for measuring the real workload of nurses (32). Lack of attention to real workload under two topics of lack of attention to nature of work in each ward and lack of attention to type of hospital activity were indicated by some participants. Choosing one general coefficient without considering the varieties of hospital beds and real workload for each bed may cause increase or decrease of manpower in hospitals. Therefore, using staff estimation standards without considering the load of services and only based on population and size of centers is inappropriate (26). 
Shivam et al. declared that human resource standards, which are only based on population ratio and standard tables, usually consider a middle number that can cause increase or decrease of resources in organizations (33). Study results showed that the lack of transparency in used methods for designing the models probably has a negative impact on accepting the model and implementing it. Issues related to subjective methods of staff estimation model has become an important problem in health in a way that policymakers are trying to use objective methods for more accurate and more reasonable estimation of human resources (34). Buchen mentioned that simplicity and clarity are one of the strengths of nursing staff estimation models used in two health systems in California and Victoria (25). The present study showed that decreased nurses' availability time, increased indirect works of nurses in recent years and outdated standards, are emphasizing the need for updating the models. They/participants believed that the improve productivity law, which was approved in 2009 by parliament and was enforced to hospitals and also the increased maternity leave from 6 to 9 months, decreased nurses' availability. Furthermore, the increased number of forms related to hospital accreditation and clinical governance programs, which are mainly on nurses, increased indirect work of nurses compared with the past. Therefore, updating the standards must be considered in different intervals. Ministry of Health and Social Services in Namibia updated used standards for staff estimation models in health and treatments centers to improve quality of health care (35). Hoi et al. concluded that, in work places that change fast, the workload must be reviewed periodically (36).

\section{Limitations}

One of the limitations of this study was that, because we could not access all the participants at once, and interview was used for data collection, it was possible that participants instead of talking about their experience used their knowledge for answering the questions. We tried by emphasizing on using their experience during interviews, thus decreasing this limitation. The other limitation was the fact that we could not access all the people who were involved in designing the current models for estimating nursing staff. Finally, this study was performed in a country with its own special culture, community, economy, and political conditions; therefore, the results could only be applicable in this environment. The amplitude of this study was limited to declaring the barriers in using nursing staff estimation models in hospitals. To properly understand the solutions for implementing these models in hospitals and also to define required features for designing proper model, further studies are required.

\section{Conclusions}

The results of present study indicate that many problems and barriers exist in designing and implementing estimated models of required nurses in hospitals. Standardization of nursing staff coefficient is announced as one of barriers in reaching the 1404 horizons by health care policymakers. On the other side, by execution of health system reform plans, The Ministry of Health wants to promote the quality of services in hospitals, in which a large part of these services are nursing care. Therefore, for designing an appropriate nursing staff estimation model and implementing it, in addition to considering the present barriers, identifying the norm required features may positively impact on norm acceptance and implementation.

\section{Acknowledgments:}

This study was a part of a Health Services Administration doctoral dissertation, which was approved by Kerman University of Medical Sciences. The authors appreciate all the participants who patiently state their experiences. This study was not funded by any institution.

\section{Conflict of Interest:}

There is no conflict of interest to be declared.

Authors' contributions:

All authors contributed to this project and article equally. All authors read and approved the final manuscript.

\section{References:}

1) Dreesch N, Dolea C, Dal Poz MR, Goubarev A, Adams O, Aregawi M, et al. An approach to estimating human resource requirements to achieve the Millennium Development Goals. Health Policy Plan. 2005; 20(5): 267-76. doi: 10.1093/heapol/czi036. PMID: 16076934.

2) Fakhri A, Aryankhesal A. The Effect of Mutual Task Sharing on the Number of Needed Health Workers at the Iranian Health Posts; Does Task Sharing Increase Efficiency? Int J Health Policy Manag. 2015; 4(8): 511-6. doi: 10.15171/ijhpm.2015.22. PMID: 26340390, PMCID: PMC4529040. 
3) Daviaud E, Chopra M. How much is not enough? Human resources requirements for primary health care: a case study from South Africa. Bull World Health Organ. 2008; 86(1): 46-51. PMID: 18235889, PMCID: PMC2647342.

4) Ministry of Health (Kenya). Norms and Standards for Health Service Delivery. Nairobi: Ministry of Health. 2006.

5) Bahadori M, Arab M, Sadeghifar J, Ahmadi B, Salimi M, Yghoubi M. Estimation of nursing staff in selected hospitals of Ilam and Ahvaz Provinces, Western Iran. Nurs Midwifery Stud. 2013; 2(2): 21725 -. PMID: 25414862, PMCID: PMC4228553.

6) Farahbakhsh M, Mostafaei M, Sadeghifar J, Estebsari F. Structure Design and Estimation of Needed Manpower for Hospital. Tehran: kamal-e-danesh. 2011.

7) Zandiyan H, GHiasvand H, Nasimi DR. Measuring inequality of distribution of health resources: A case study. Payesh. 2012; 11(6): 799-805.

8) Sadeghifar J, Pourreza A, Ahmadi B, Zeraati H, Arab M. Assessment of necessary staff for hospitals of Ilam university of medical sciences in accordance with personnel criteria and standards of Iranian health ministry. JIUM. 2011; 19(1): 24-31.

9) Ghazi Mirsaeid J, Mirzaie M, Haghshenas E, Dargahi H. Human resources distribution among tehran university of medical sciences hospitals. Payavard Salamat. 2014; 7(5): 432-46.

10) Sherman RO, Chiang $\neg$-Hanisko L, Koszalinski R. The ageing nursing workforce: a global challenge. J Nurs Manag. 2013; 21(7): 899-902. doi: 10.1111/jonm.12188.

11) Dehghan-Nayeri N, Ghaffari F, Shali M. Exploring Iranian nurses' experiences of missed nursing care: a qualitative study: a threat to patient and nurses' health. Med J Islam Repub Iran. 2015; 29: 276. PMID: 26793667, PMCID: PMC4715394.

12) Hendrich A, Chow MP, Skierczynski BA, Lu Z. A 36-hospital time and motion study: how do medicalsurgical nurses spend their time? Perm J. 2008; 12(3): 25-34. PMID: 21331207, PMCID: PMC3037121.

13) Hassmiller SB, Cozine M. Addressing the nurse shortage to improve the quality of patient care. Health Aff (Millwood). 2006; 25(1): 268-74. doi: 10.1377/hlthaff.25.1.268. PMID: 16403763.

14) Needleman J, Buerhaus P, Pankratz VS, Leibson CL, Stevens SR, Harris M. Nurse staffing and inpatient hospital mortality. N Engl J Med. 2011; 364(11): 1037-45. doi: 10.1056/NEJMsa1001025. PMID: 21410372.

15) Farsi Z, Dehghan-nayeri N, Negarandeh R, Broomand S. Nursing profession in Iran: an overview of opportunities and challenges. Jpn J Nurs Sci. 2010; 7(1): 9-18. doi:10.1111/j.1742-7924.2010.00137.x. PMID: 20618672.

16) Zarea K, Negarandeh R, Dehghan-Nayeri N, Rezaei-Adaryani M. Nursing staff shortages and job satisfaction in Iran: Issues and challenges. Nurs Health Sci. 2009; 11(3): 326-31. doi: 10.1111/j.14422018.2009.00466.x. PMID: 19689643.

17) Rossetti AC, Gaidzinski RR. Estimating the nursing staff required in a new hospital. Rev Lat Am Enfermagem. 2011; 19(4): 1011-7. doi:10.1590/S0104-11692011000400021. PMID: 21876955.

18) Sigarodi A D-NN, Rahnavard Z, Norisaeed A. Qualitative research methodology: phenomenology. Holist Nurs Midwifery. 2012; 22(2): 56-63.

19) Adib hajbagheri M, Parvizi S, Salsali S. Qualitative Research Methods. Boshry Pubilshing. 2011.

20) Ghada Abu Shosha. Employment of colaizzi's strategy in descriptive phenomenology: a reflection of a researcher. Euro Sci J. 2012; 8(27): 31-43

21) Anderson C. Presenting and evaluating qualitative research. Am J Pharm Educ. 2010; 74(8): 141. PMID: 21179252, PMCID: PMC2987281.

22) Morse JM, Barrett M, Mayan M, Olson K, Spiers J. Verification strategies for establishing reliability and validity in qualitative research. IJQM. 2002; 1(2): 13-22.

23) Biglar M, Bastani P, Soudabeh Vatan khah. The Challenges Of Stewardship In Medical Education System: A Qualitative Approach. Payavard Salamat. 2013; 7(4): 299-311.

24) Lunenburg FC. The Decision-Making Process in an Organization. IJBAM. 2011; 15(1): 1-9

25) Buchan J. A certain ratio? The policy implications of minimum staffing ratios in nursing. J Health Serv Res Policy. 2005; 10(4): 239-44. doi: 10.1258/135581905774414204. PMID: 16259691.

26) Mugisha JG, Namaganda G. Using the Workload Indicator of Staffing Needs (WISN) methodology to assess work pressure among the nursing staff of Lacor Hospital. Health Pol Dev. 2008; 6(1): 1-15.

27) Sadeghifar J, Tofighi Sh, Hasani M, RekabEslami S, Raadabadi M, Moosavi SM. Estimation of the Necessary Nursing Manpower in Selected Hospital Affiliated with Jundishapur University of Medical Sciences. Sci Med J Ahwaz Jundishapur Univ Med Sci. 2013; 5(1): 1-8. 
28) Reiter KL, Harless DW, Pink GH, Mark BA. Minimum nurse staffing legislation and the financial performance of California hospitals. Health Serv Res. 2012; 47(3pt1): 1030-50. doi: 10.1111/j.14756773.2011.01356.x. PMID: 22150627, PMCID: PMC3337946.

29) The sixth draft development plan, [online]. [Cited 2016 April 24]. Available from: http://www. budget.farhang.gov.ir/fa/peshnvesbrnameh6/peshnvesbrnameh63

30) Jajarmizadeh M, Forozandeh-Dehkord L, Abasi N, Tadauon A. Native Model of Human Resource Management with on Emphasis on Contextual Factor of the Administrative System. Management Research. 2015; 7(26): 45-62.

31) Ignjatović M, Svetlik I. European HRM clusters. EBS review. 2003; 17(3): 25-39.

32) Twigg D, Duffield C. A review of workload measures: a context for a new staffing methodology in Western Australia. Int J Nurs Stud. 2009; 46(1): 131-9. doi:10.1016/j.ijnurstu.2008.08.005. PMID: 18789439.

33) Shivam S, Roy RN, Dasgupta S, Das Bhattacharyya K, Misra RN, Roy S, et al. Nursing Personnel Planning for Rural Hospitals in Burdwan District, West Bengal, India, Using Workload Indicators of Staffing Needs. J Health Popul Nutr. 2014; 32(4): 658-64. PMID: 25895199 , PMCID: PMC4438696.

34) Vali L, Tabatabaee SS, Kalhor R, Amini S, Kiaei MZ. Analysis of Productivity Improvement Act for Clinical Staff Working in the Health System: A Qualitative Study. Glob J Health Sci. 2015; 8(2): 106-16. doi:10.5539/gjhs.v8n2p106. PMID: 26383203, PMCID: PMC4803926.

35) McQuide PA, Kolehmainen-Aitken RL, Forster N. Applying the workload indicators of staffing need (WISN) method in Namibia: challenges and implications for human resources for health policy. Hum Resour Health. 2013; 11(1): 64. doi: 10.1186/1478-4491-11-64. PMID: 24325763, PMCID: PMC4028865.

36) Hoi SY, Ismail N, Ong LC, Kang J. Determining nurse staffing needs: The workload intensity measurement system. J Nurs Manag. 2010; 18(1): 44-53. doi: 10.1111/j.1365-2834.2009.01045.x. PMID: 20465728. 